\title{
Catecholaminergic Polymorphic Ventricular Tachycardia. An Important Diagnosis in Children With Syncope and Normal Heart
}

\author{
Luiz Roberto Leite, Kleber R. Ponzi Pereira, Sílvio R. B. A lessi, Angelo A. V. de Paola \\ São Paulo, SP - Brazil
}

\begin{abstract}
Syncope in children is primarily related to vagal hyperreactivity, but ventricular tachycardia (VT) way rarely be seen. Catecholaminergic polymorphic VT is a rare entity that can occur in children without heart disease and with a normal QT interval, which may cause syncope and sudden cardiac death. In this report, we describe the clinical features, treatment, and clinical follow-up of three children with syncope associated with physical effort or emotion and cathecolaminergic polymorphic VT. Symptoms were controlled with beta-blockers, but one patient died suddenly in the fourth year of follow-up. Despite the rare occurrence, catecholaminergic polymorphic VT is an important cause of syncope and sudden death in children with no identified heart disease and normal QT interval.
\end{abstract}

Syncope in children is primarily related to vagal hyperreactivity ${ }^{1,2}$. In this population, ventricular tachycardia(VT) is rarely seen, and its occurrence is often related to congenital cardiac abnormalities. Although ventricular tachyarrhythmias in the absence of structural heart disease are benign, some arrhythmias must be clearly recognized as life threatening. Catecholaminergic polymorphic ventricular tachycardia is a rare entity that can occur in children without heart disease and with a normal QT interval, which may cause syncope and sudden cardiac death ${ }^{3-6}$. The purpose of the present report is to describe the clinical features, treatment, and clinical follow-up of children with syncope and catecholaminergic polymorphic ventricular tachycardia.

Between January 1989 and July 1999, three children with syncope and catecholaminergic polymorphic VT were followed at the division of clinical cardiac electrophysiology

São Paulo Federal University - Paulista School of Medicine

Mailing address: Angelo A. V. de Paola - Setor de Eletrofisiologia Clínica Napoleão de Barros, 593 - 04024-002 - São Paulo, SP - Brazil - E-mail: eletrof@uol.com.br at São Paulo Hospital. The clinical presentation, 12-lead ECG pattern, 24-hour Holter monitoring, treadmill test (Bruce protocol), isoproterenol infusion, tilt-table test, echocardiography, left ventricular angiography, and invasive electrophysiologic study were evaluated (table I). Patients were treated with medical therapy, and evaluated monthly in the first 6 months and every two months in the first year. Clinical evaluation, Holter monitoring, treadmill testing, and isoproterenol infusion were performed at each appointment.

\section{Case Reports}

Case 1 - A 10-year-old girl experienced her first syncope episode during emotional stress when she was seven years old. She suffered many similar episodes thereafter, which were always triggered by physical effort or emotion. Her initial diagnosis was epilepsy, and she was treated with barbiturates. She was referred to our hospital after being resuscitated from sudden cardiac death. During the diagnostic evaluation, 24-hour Holter monitoring showed a sequence of isolated premature ventricular complexes, which degenerated into sustained polymorphic ventricular tachycardia (bidirectional), leading to syncope and convulsive movements with spontaneous recovery (figure 1). Treadmill testing (Bruce protocol) and isoproterenol infusion at increasing doses were able to reproduce sustained polymorphic VT. During treadmill testing, Holter monitoring, and isoproterenol infusion the first premature ventricular complex (PVC) started when her heart rate exceeded 100-120 beats/ min, which were followed by bigeminy, coupled PVC, and nonsustained VT. Syncope occurred only during Holter monitoring (figure 1).

After the diagnosis of catecholaminergic polymorphic VT, propanolol was started, which was effective in abolishing episodes of syncope, as well as ventricular tachycardia on Holter monitoring, treadmill testing, and isoproterenol infusion. Although the patient had remained asymptomatic during follow-up, she experienced nonsustained poly- 


\begin{tabular}{|c|c|c|c|c|c|c|c|c|c|}
\hline & Age & Sex & Basal & QTc & U wave & ECO & Iso/TT & PVS & Clinical Follow-up \\
\hline Case 1 & 10 & $\mathrm{~F}$ & 54 & 380 & Presente & Normal & Poly VT & Not Induced & $\begin{array}{l}\text { Sudden death } \\
48 \text { months }\end{array}$ \\
\hline Case 2 & 8 & $\mathrm{~F}$ & 68 & 400 & Presente & Normal & Poly VT & Not Induced & $\begin{array}{l}\text { Asymptomatic } \\
24 \text { months }\end{array}$ \\
\hline Case 3 & 12 & $\mathrm{~F}$ & 58 & 360 & Presente & Normal & Poly VT & Poly VT & $\begin{array}{l}\text { Asymptomatic } \\
8 \text { months }\end{array}$ \\
\hline
\end{tabular}

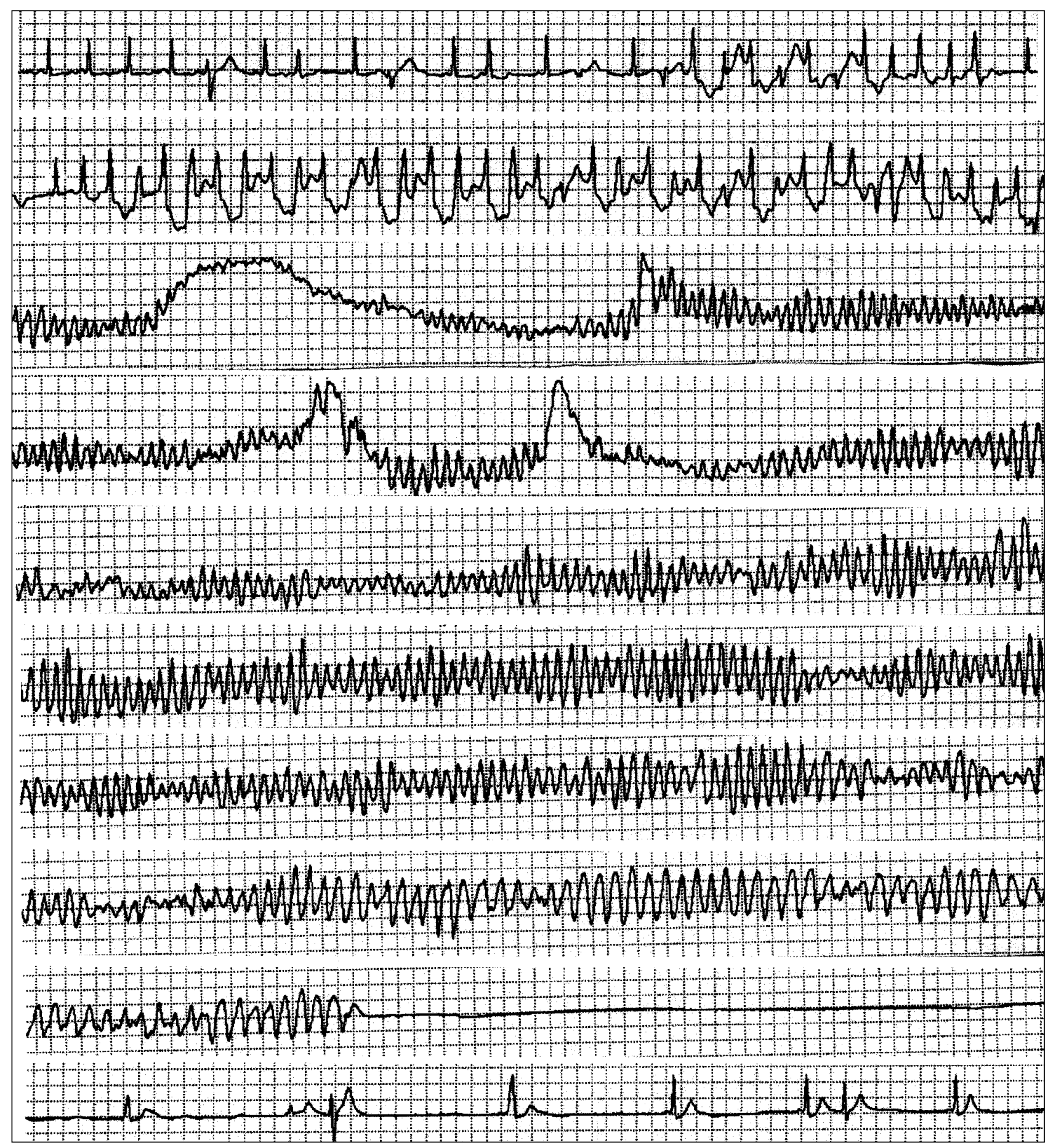

Fig. 1 - Holter recording (V1 lead) during a syncopal episode and convulsion. Tracing shows polymorphic VT initiation preceded by isolated VEB and nonsustained VT. 
morphic VT on Holter monitoring, probably related to her increasing age, which should lead to an increase in propranolol doses. She died suddenly in the fourth year of follow-up after having discontinued her outpatient visits for 8 months.

Case 2 - An eight-year-old female patient was referred to us with a history of frequent near-syncope and three syncope episodes in an 18-month period, which were always related to exercise or emotion. She had been followedup in another hospital with a diagnosis of vagal hyperreactivity, and was off drug therapy. The clinical investigation showed induction of polymorphic VT when sinus tachycardia achieved 120 beats/min during treadmill testing and isoproterenol infusion. Short runs of supraventricular tachycardia between polymorphic VT were also observed (figure 2). The tilt-table test was negative.

She has been treated with nadolol, and no further episodes of syncope have occurred. Serial exercise tests were performed, and no arrhythmia was induced.

Case 3 - A 12-year-old female patient was referred to our institution because of several episodes of syncope over the previous five years. She had suffered four episodes in the prior 6 months. A familial history revealed sudden cardiac death of her brother at the age of 6 . She had been treated for epilepsy with carbamazepine. Her physical examination was unremarkable. The tilt-table test was negative. Polymorphic VT was induced during treadmill testing (Bruce protocol), Holter monitoring, and isoproterenol infusion, and a diagnosis of catecholaminergic polymorphic VT was made. The electrophysiologic study induced polymorphic VT (figure 3) by programmed ventricular stimulation, associated with loss of consciousness and need of electric defibrillation, which converted the arrhythmia to polymorphic VT of lower frequency followed by spontaneous termination (Figure 4). Treatment with nadolol was successful, and VT episodes were abolished in Holter monitoring, treadmill testing, and isoproterenol infusion. After 8 months of follow-up, polymorphic VT was induced only by isoproterenol infusion. However, the child has remained asymptomatic during clinical follow-up without VT either on Holter monitoring or treadmill testing.

\section{Discussion}

Despite of the rare occurrence, catecholaminergic polymorphic VT is an important cause of syncope in children with no identified heart disease and normal QT interval.

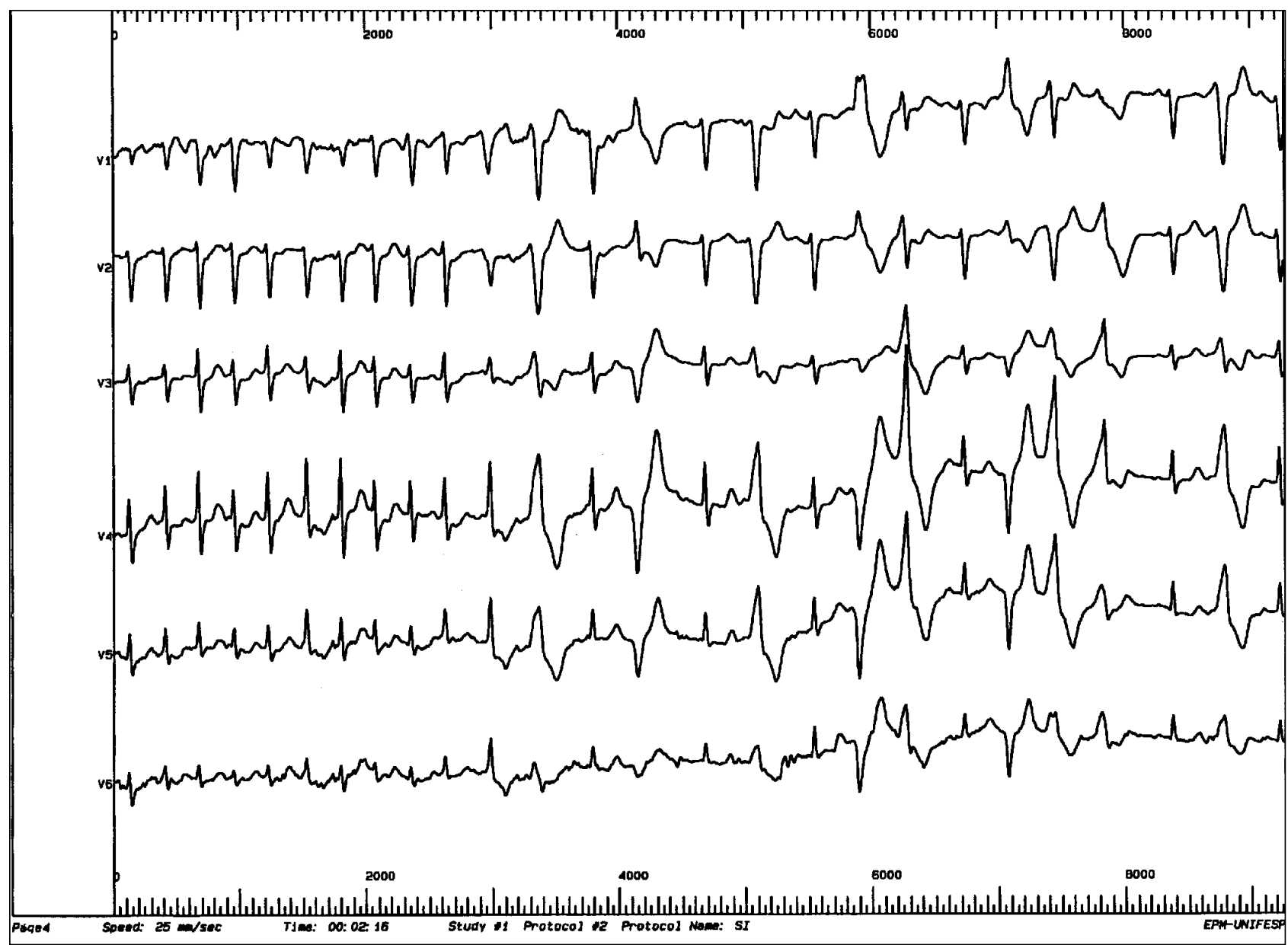

Fig. 2 - ECG recording during isoproterenol infusion. Supraventricular tachycardia between episodes of polymorphic VT. Leads V1 to V6. 


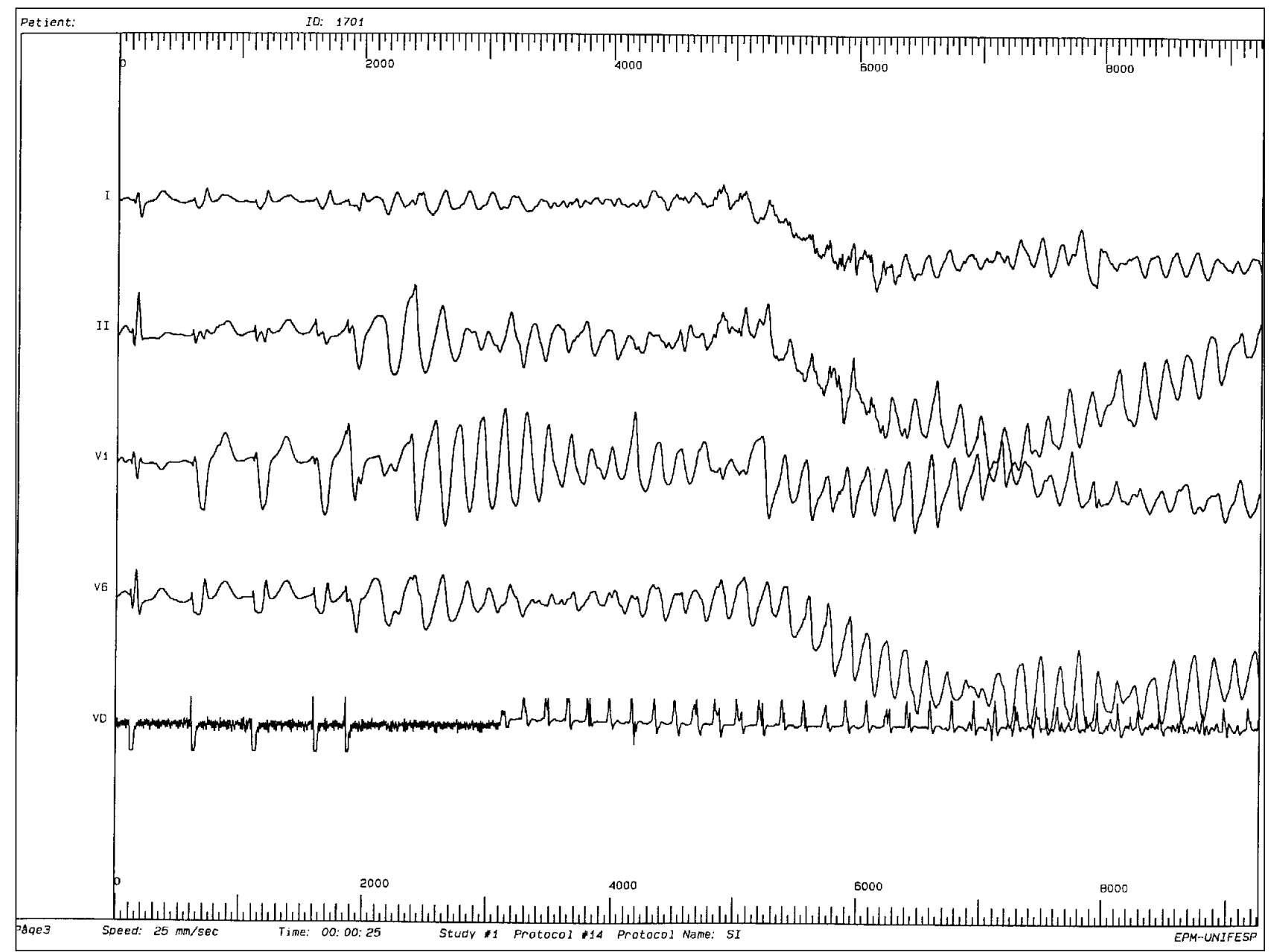

Fig. 3 - Programmed ventricular stimulation (S1-S1=500ms, S1-S2=250ms), inducing polymorphic VT during electrophysiological study with hemodynamic collapse. Leads D1, $\mathrm{D} 2, \mathrm{~V} 1, \mathrm{~V} 6$, and right ventricle.

Although this arrhythmia has been clearly defined for over two decades ${ }^{3}$, only 60 cases have been reported in the literature. Syncope was the most common symptom described. Therefore, physicians must remember the possibility of catecholaminergic polymorphic VT whenever syncope associated with physical effort or emotion occurs in young patients, especially when the QT interval is normal.

Many cases such as those reported in the present study, in the absence of heart disease but with a normal QT interval, may be misdiagnosed as epilepsy. In a series of 21 cases, Leenhardt et al ${ }^{6}$ reported that $50 \%$ of patients had been previously treated as epileptics. In that study, a correct diagnosis was made 24 months after the onset of symptoms. The present study also demonstrated a significant delay before the correct diagnosis (mean time of 38 months) was made. Due to its potential lethal outcome, suspicion of catecholaminergic polymorphic VT in children with physical and emotional syncope is mandatory.

Although the genetic and molecular substrate of catecholaminergic polymorphic VT has not yet been defined, the genetic origin of this arrhythmia is confirmed by familial history of syncope or sudden death in $40 \%$ of cases ${ }^{6}$. In our study, one of the 3 children had a familial history of sudden cardiac death. Due to the low penetrance of congenital long QT syndrome ${ }^{7}$, it is possible that catecholaminergic polymorphic VT may be a variant of this entity. Further research using new technologies is necessary to confirm this hypothesis.

The features in 12-lead ECG are nonspecific. Relative sinus bradycardia and the presence of $U$ waves are the only characteristic findings on $\mathrm{ECG}^{8}$.

The mechanism and electrophysiological substrate for catecholaminergic polymorphic VT has not been clearly identified. Leenhardt et al ${ }^{6}$ suggested that triggered activity may be the involved mechanism. Later, Nakagima et al ${ }^{9}$ using monophasic action potential recordings before and during pharmacological intervention (isoproterenol and beta-blockers) in one patient with catecholaminergic polymorphic VT, have related that triggered activity due to delay after depolarization was probably the electrophysiological mechanism responsible for this arrhythmia.

In our study, programmed ventricular stimulation induced polymorphic VT in one patient. Horowitz et al ${ }^{10}$ induced Torsades de Pointes during programmed ventricular stimulation in 19 of 21 patients with structural heart disease and previous episodes of this arrhythmia. Metabolic distur- 


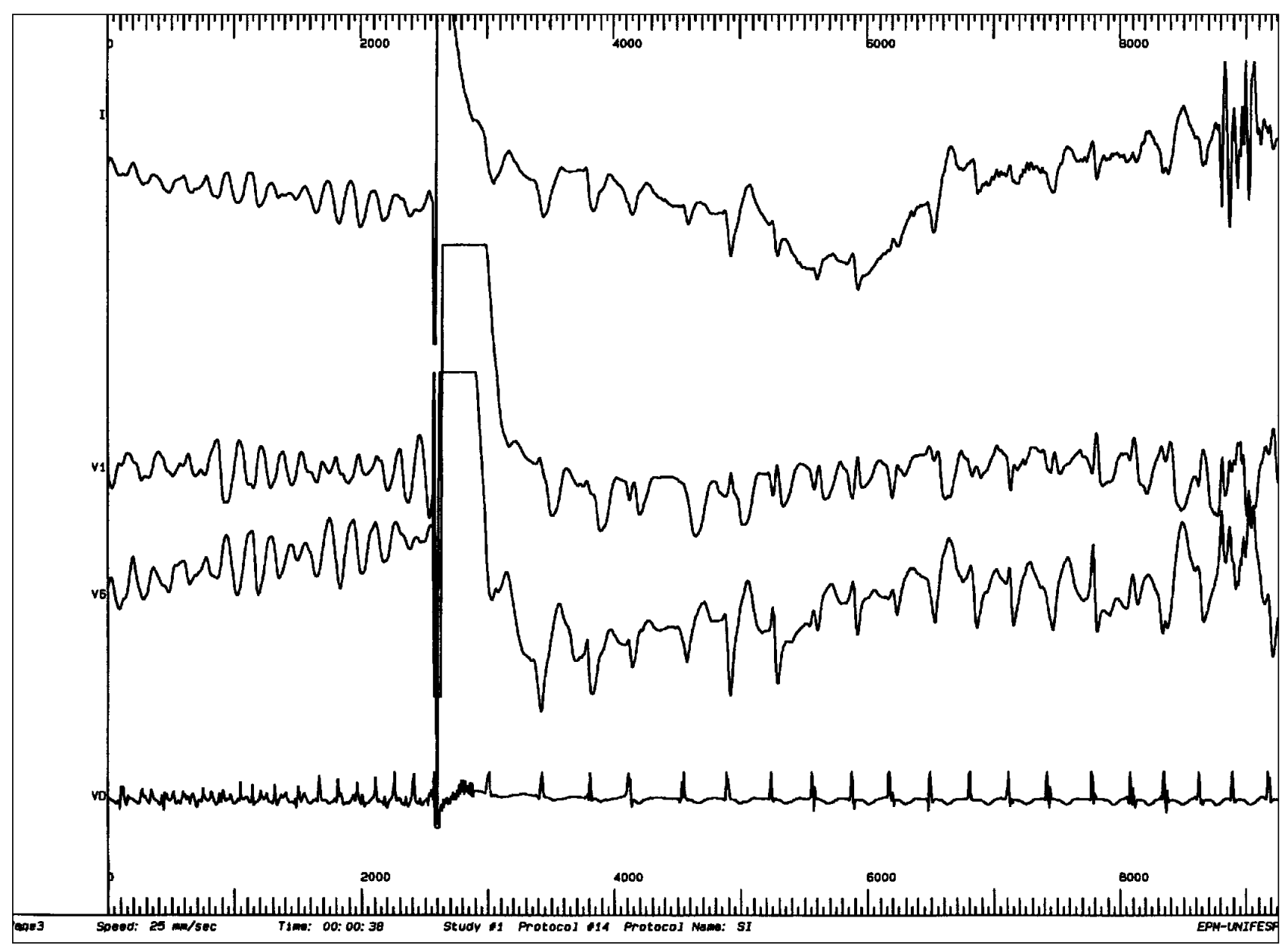

Fig. 4-Same patient as that of figure 3: After electric defibrillation (300J), VT degenerated to VT with a lower cycle length.

bances or reversible causes of polymorphic VT were ruled out in that study. Although induction of tachyarrhythmias by programmed ventricular stimulation was not frequently reported in catecholaminergic polymorphic VT, it might be compatible with the suggested triggered mechanism.

Beta-blockers are the treatment of choice for catecholaminergic polymorphic VT. Although no differences exist among various agents, we and other authors have preferred nadolol because of its prolonged half-life. Doses must be titrated to carefully prevent the heart rate from exceeding 130 beats per minute ${ }^{6}$. Because in any situation patients are usually growing up, doses must be adjusted by $\mathrm{kg} /$ weight. Other antiarrhythmic drugs such as amiodarone and class I drugs have proved to be ineffective ${ }^{6}$.

Classically, the success of treatment can be assessed by the disappearance of symptoms and the absence of VT on Holter recordings and exercise tests ${ }^{4-6}$. Isoproterenol infusion was also used in our patients to reproduce increased adrenergic discharge. However, the value of this method for evaluation of therapeutic efficacy is not completely defined. In patients who achieved clinical therapy efficacy, it was usual to observe PVC or even periods of nonsustained VT on clinical follow-up.
Sudden death can occur in $50 \%$ of the cases before the age of 20 in patients not treated with beta-blockers ${ }^{6}$. Four deaths have been reported among patients treated with beta-blockers. In one case, the patient forgot to take the drug on the day of the event. In another, the death occurred in the context of viral myocarditis, and in two others under nonspecific circumstances. Our patient was taking propranolol regularly, but we could not confirm whether she took the medication on the day of death.

Because beta-blockers are highly effective in patients with catecholaminergic polymorphic VT, nonmedical treatment such as implantable cardioverter defibrillation is not mandatory.

\section{Conclusion}

Although vagal hyperreactivity is a major cause of syncope in the pediatric population, catecholaminergic polymorphic VT must be individualized in children without heart disease but with a normal QT interval, especially when episodes of syncope are related to physical effort or emotion. Delay in diagnosis and inadequate therapy can lead to sudden cardiac death. 


\section{References}

1. Ruckman RN. Cardiac causes of syncope. Pediatr Rev 1987;9: 101-8.

2. Pratt JL, Fleisher GR. Syncope in children and adolescents. Pediatr Emerg Care 1989; 5: 80-2.

3. Reid DS, Tynan M, Braidwood L, Fitzgerald GR. Bidirectional tachycardia in a child. A study using His bundle electrography. Br Heart J 1975; 37: 339-44.

4. De Paola AA, Horowitz LN, Marques FB, et al. Control of multiform ventricular tachycardia by propranolol in a child with no identifiable cardiac disease and sudden death. Am Heart J 1990; 119: 1429-32.

5. Eisenberg SJ, Scheinman MM, Dullet NK, et al. Sudden cardiac death and polymorphous ventricular tachycardia in patients with normal QT intervals and normal systolic cardiac function. Am J Cardiol 1995; 75: 687-92.

6. Leenhardt A, Lucet V, Denjoy I, Grau F, Ngoc DD, Coumel P. Catecholaminergic polymorphic ventricular tachycardia in children. A 7-year follow-up of 21 patients. Circulation 1995; 91: 1512-9.
7. Priori SG, Napolitano C, Schwartz PJ. Low penetrance in the long-QT syndrome: clinical impact. Circulation 1999; 99: 529-33.

8. Noh CI, Gillette PC, Case CL, Zeigler VL. Clinical and electrophysiological characteristics of ventricular tachycardia in children with normal hearts. Am Heart J 1990; 120: 1326-33.

9. Nakajima T, Kaneko Y, Taniguchi Y, Hayashi K, Takizawa T, Suzuki T, Nagai R. The mechanism of catecholaminergic polymorphic ventricular tachycardia may be triggered activity due to delayed afterdepolarization. Eur Heart J 1997; 18: 530-1.

10. Horowitz LN, Greenspan AM, Spielman SR, Josephson ME. Torsades de Pointes: electrophysiologic studies in patients without transient pharmacologic or metabolic abnormalities. Circulation 1981; 63: 1120-7. 